\title{
LUCHA CONTRA EL PLAGIO DESDE LAS BIBLIOTECAS UNIVERSITARIAS
}

\author{
María-Isabel Domínguez-Aroca
}

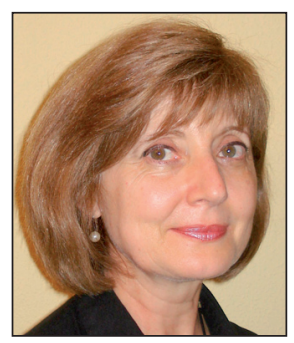

María-Isabel Domínguez-Aroca es licenciada en historia por la Universidad de Alcalá (UAH), profesora de magisterio por la Universidad de Valencia (UV) y documentalista especializada por la Universidad Complutense de Madrid (UCM). Actualmente es jefa de las bibliotecas del Área de Ciencias y Ciencias de la Salud de la UAH. Representante por Rebiun (Red de Bibliotecas Universitarias) en el grupo de trabajo de alfin (afabetización informacional) del Consejo de Cooperación Bibliotecaria del Ministerio de Cultura de 2007 a 2011, período en el que ha participando en la elaboración de los contenidos del portal Alfared, especialmente en el apartado de bibliotecas universitarias.

Universidad de Alcalá. Facultad de Medicina Ctra. Madrid-Barcelona, Km. 33,6 28871 Alcalá de Henares (Madrid), España misabel.dominguez@uah.es

\section{Resumen}

Internet se ha convertido en la fuente principal donde localizar información, lo que ha propiciado entre el alumnado universitario la práctica de plagio y en concreto de ciberplagio. El panorama académico está abonado para el plagio y otras prácticas deshonestas, por lo que cada vez más bibliotecas universitarias españolas -aunque de forma desigual- estén incorporando información legal, didáctica, programas antiplagio, etc., en sus webs. Esta decisión puede ser estratégica si la enmarcamos en las nuevas metodologías docentes del EEES en las que el alumnado debe realizar un gran número de trabajos, y en la cada vez mayor implementación en las universidades españolas de las competencias informacionales.

\section{Palabras clave}

Plagio académico, Bibliotecas universitarias, Competencias informacionales, Alfin, Cl2, Educación superior.

\section{Title: Fighting against plagiarism from university libraries}

\begin{abstract}
The internet has become the main source where information can be located, which has facilitated certain university student practices, plagiarism and cyber-plagiarism in particular. Academic plagiarism and related dishonest practices result in the incorporation, although unevenly, of legal, educational, and anti-plagiarism software, etc., on websites in an increasing number of Spanish university libraries. This can be a strategic decisión if considered within the framework of the new EHEA teaching methods, which require that students produce a large number of academic papers, and the growing implementation in the Spanish universities of informational skills.
\end{abstract}

\section{Keywords}

Academic plagiarism, University libraries, Information literacy, Higher education, Cyberplagiarism.

Domínguez-Aroca, María-Isabel. "Lucha contra el plagio desde las bibliotecas universitarias". El profesional de la información, 2012, septiembre-octubre, v. 21, n. 5, pp. 498-503.

http://dx.doi.org/10.3145/epi.2012.sep.08

\section{Introducción}

Internet se ha convertido para el alumno universitario en la fuente principal donde localizar información (Sureda-Negre; Comas-Forgas, 2006). Nueve de cada diez estudiantes la utilizan para documentarse (Six Degrés, 2008) y además el universitario en general cree que posee habilidades muy altas para usarla y documentarse (Salaway; Caruso, 2008), mientras que no se siente competente en el uso de la biblioteca (Comas-Forgas et al., 2011a).

Artículo recibido el 01-07-12

Aceptación definitiva: 11-07-12
Las facilidades que presentan las nuevas tecnologías para copiar y pegar hacen del ciberplagio uno de los tipos de plagio más usual. Cavanillas (2008) cree que éste debería estar recogido en la normativa universitaria, y que requiere al menos dos políticas complementarias: la educación de los estudiantes, para que sepan de forma clara qué es y qué no es ciberplagio, y la formación del profesorado, para que lo conceptúe de modo uniforme.

La Real Academia Española (RAE) define el plagio como "co- 


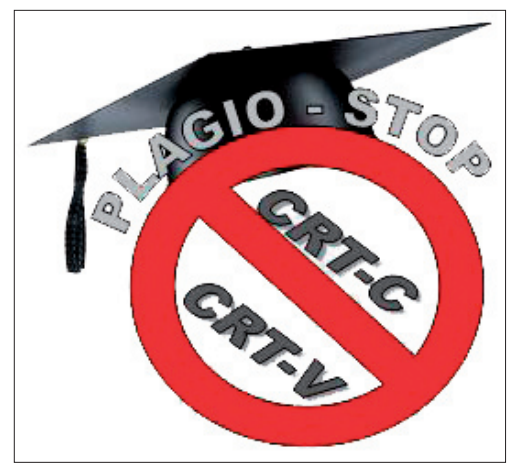

piar en lo sustancial obras ajenas, dándolas como propias", algo que se realiza en la actualidad de forma indiscriminada al copiar textos procedentes de internet. Podríamos definir el ciberplagio como: "aquellos materiales presentados como fruto del trabajo personal que, en realidad, han sido copiados parcial o totalmente mediante procedimientos informáticos, sin mencionar su autoría original; la procedencia de los mismos es mayoritariamente la red internet, si bien puede tratarse también de copia de otras fuentes, como enciclopedias en cd-rom, o trabajos de compañeros (UrbinaRamírez, 2004). No siempre es fácil de detectar, a pesar de la existencia de un número cada vez mayor de programas antiplagio gratuitos o de pago.

\section{El plagio es una erosión de los principios éticos de honestidad académica, esfuer- zo personal, seriedad y profundidad en el proceso de aprendizaje y descubri- miento científico}

El plagio es una erosión entre otros de los principios éticos de honestidad académica (Blanch-Mur; Rey-Abella; FolchSoler, 2006), esfuerzo personal, seriedad y profundidad en el proceso de aprendizaje y descubrimiento científico. Nos empobrece como personas, es un acto egoísta que cercena la creatividad e innovación del ser humano (que debe ser responsable de la veracidad y calidad de su trabajo), y además puede entrañar responsabilidades penales o sanciones académicas, pero sobre todo supone vulnerar los derechos morales relacionados con la autoría, una de las partes más importantes de los derechos de autor.

Es también una infracción del derecho de autor sobre cualquier tipo de obra, al presentar la copia como propia, sin autorización expresa de la persona que la creó, sea dueña de la misma o posea los derechos sobre ella. Es una doble infracción pues supone "robar al autor y engañar al destinatario de la obra plagiada" (Cavanillas, 2008).

La normativa fundamental española sobre plagio académico se fundamenta en:

- Ley de propiedad intelectual, regulada por el Real decreto legislativo 1/1996, de 12 de abril (modificada por la Ley 5/1998 de 12 de abril). En su artículo 1 dispone que "la propiedad intelectual de una obra literaria, artística o científica corresponde al autor por el solo hecho de su creación".

- Ley orgánica 2/1984 de 26 de marzo que regula el Derecho de rectificación: "Toda persona, natural o jurídica, tiene derecho a rectificar la información difundida, por cualquier medio de comunicación social, de hechos que le aludan, que considere inexactos y cuya divulgación pueda causarle perjuicio".

- Código penal: Ley orgánica 10/1995 de 23 de noviembre. Establece que constituye delito "la reproducción, distribución, comunicación pública o plagio de obras protegidas por la propiedad intelectual, con ánimo de lucro y en perjuicio de terceros, sin autorización de sus legítimos titulares" (art. 270).

- Normativa propia de cada universidad. Por ejemplo, en el caso de la Universidad de Alcalá queda recogido en sus Estatutos aprobados en la sesión del Claustro de 14 de mayo de 2003, modificados en la sesión del 20 de octubre de 2003 y por el Real Decreto 18/2012, de 26 de enero, del Consejo de Gobierno (BOCM n. 35 de 10 de febrero de 2012). En el artículo 137, en el apartado de derechos de los estudiantes, dice: “...a la propiedad intelectual y los derechos de autor, en virtud de lo cual nadie podrá usar sin su consentimiento sus trabajos, estudios, ensayos y otras realizaciones, de acuerdo con lo que establece la legislación vigente".

Algunas bibliotecas universitarias (BUs) han incorporado esta normativa a sus webs, aunque aún no es una práctica muy extendida, y existe un gran número de ellas sin ninguna información sobre el plagio. Sin embargo muchas sí proporcionan información sobre el uso ético de la información, o sobre propiedad intelectual durante la formación en competencias en información (alfin); o como ocurre en muchas más ocasiones, publican normativa sobre citas bibliográficas y estilos de cita en la web de la biblioteca.

\section{Plagio y prácticas deshonestas del universitario español}

La forma más común de ciberplagio es el "copia y pega" o el conocido "control-c, control-v", algo bastante popular en el entorno académico como lo demuestran distintos estudios sobre ello, aunque no sean muchos en España con respecto a los realizados en los países anglosajones.

Las acciones académicamente incorrectas relativas a la elaboración y presentación de trabajos académicos se pueden resumir siguiendo a Comas-Forgas et al. (2011b) en:

- copiar de webs u otros recursos de la Red documentos enteros o fragmentos y entregarlos como trabajos propios;

- copiar fuentes impresas;

- copiar sin citar fragmentos o documentos enteros de libros o de revistas;

- copiar partes de trabajos entregados en años anteriores y entregarlos como nuevos, tanto si es propio como si es ajeno;

- entregar como propio un trabajo realizado por otro alumno en años anteriores;

- elaborar un trabajo para que lo entregue otra persona;

- comprar o vender trabajos académicos;

- falsear bibliografía, datos, resultados o recursos en los trabajos académicos;

- colaborar en la elaboración de un trabajo sin estar permitido.

Las prácticas deshonestas han sido analizadas mediante el uso de encuestas con muestras más o menos amplias de 
alumnos. Ejemplos destacados son el estudio de la Universitat de les Illes Balears a alumnos pregraduados durante el curso 2009-2010 (Comas-Forgas; Sureda-Negre; Oliver-Trobat, 2011); y el de la Universidad de Alicante a alumnos del grado de Relaciones Laborales y Recursos Humanos comparándolo con el estudio de Sureda-Negre y Comas-Forgas de 2008 , con resultados alarmantes, pues muestra que el $90 \%$ de los alumnos había realizado alguna de las formas de ciberplagio (Beléndez-Vázquez et al., 2011).

Otros estudios son los llevados a cabo en 2009 en la Facultad de Ciencias de la Información de la Universidad Complutense de Madrid (Caldevilla-Domínguez, 2010a); en la Universitat de les Illes Balears a alumnos de pregrado de todas las titulaciones (Sureda-Negre; Comas-Forgas, 2008); el realizado a nivel nacional a estudiantes registrados en el portal Universia (en el que casi un $60 \%$ de los alumnos manifestaba haber realizado ciberplagio) (Sureda-Negre; Morey, 2008); y un estudio más antiguo al alumnado de la Escuela Universitaria de Enfermería, Fisioterapia y Nutrición de la Universitat Ramon Llull (Blanch-Mur; Rey-Abella; FolchSoler, 2006). Todos ponen de manifiesto que prácticamente siete de cada diez alumnos encuestados admiten haber copiado fragmentos de textos o textos completos al menos una vez durante sus estudios universitarios.

Junto con el ciberplagio no hay que olvidar la existencia de otras prácticas como:

- copia de fuentes impresas;

- cita de referencias bibliográficas no consultadas;

- falsear datos para resolver un problema o inventar datos en una investigación, etc.

Las justificaciones más habituales del alumnado para copiar de internet, siguiendo a Comas-Forgas et al. (2009) e incorporando aportaciones propias, podrían resumirse en: obtención de mejores calificaciones académicas; pereza; facilidad y comodidad y anonimato para acceder a internet; mala gestión del tiempo dedicado al estudio y a la elaboración de los trabajos académicos; desconocimiento de las pautas básicas para la elaboración de un trabajo académico; exceso de demanda de trabajos con la nueva metodología del EEES (Beléndez-Vázquez et al., 2011), creencia de que muchos trabajos no los leerá el profesor por falta de tiempo; escasa valoración respecto a la nota media de la asignatura. Aluden también a la desmotivación al saber que compañeros han "colado" trabajos plagiados con poco esfuerzo y han conseguido buenas calificaciones y obtener peores resultados por trabajos propios que han necesitado mucho más tiempo.

Una de las causas de cometer plagio es la falta de formación para la realización de trabajos académicos

Posibles razones para cometer plagio son las siguientes:

- Decadencia de la cultura del esfuerzo en nuestra sociedad actual.

- Falta de formación en muchos estudios sobre metodología para la realización de trabajos académicos (en Uni- versia, el $42 \%$ reconocía no haber recibido este tipo de formación) (Sureda-Negre; Comas-Forgas, 2008). Tal vez sea ésta una de las razones por la que algunas bibliotecas universitarias elaboran material de apoyo que lo explica. A modo de ejemplo podemos visitar el portal Alfared donde junto a tutoriales de autoformación extranjeros, existen algunos recursos formativos realizados por BUs españolas sobre competencias informacionales.

http://www.alfared.org/page/bibliotecas-universita rias $/ 641$

- Poca claridad en las instrucciones dadas a los alumnos por parte del docente cuando solicita los trabajos.

- Insuficiente difusión por parte del profesorado de los recursos con los que cuenta la biblioteca para localizar información de calidad.

- Complejidad para localizar información en los recursos de la biblioteca frente a la facilidad de Google.

- Desconocimiento de los estilos de cita por parte del alumnado y en algunos casos, falta de exigencia del profesorado en la realización de las referencias bibliográficas de forma correcta al presentar un trabajo académico.

- Escasa o ninguna formación en competencias informacionales del alumnado universitario a pesar de los esfuerzos de muchas BUs para que éstas se implementen (Crue/Rebiun, 2011).

Ante esto, las medidas más implementadas para luchar contra el plagio son los programas informáticos como Antiplagiarist, Articlechecker, Compilatio, Copionic, Copycatch, Copyscape, Crosscheck, iThenticate.com, Plagium, Turnitin. com, Viper, etc., aunque los últimos resultados sobre su uso no son muy satisfactorios, pues incluso parecen haber provocado nuevas prácticas fraudulentas (Sureda-Negre; Comas-Forgas, 2008).

Es importante que los alumnos consigan habilidades informacionales que les permitan sacar el máximo provecho tanto de la información suscrita por la biblioteca, como de la accesible en internet, evaluándola de forma crítica y realizando un uso ético de la misma. En definitiva, se debe lograr que el alumno respete la propiedad intelectual de quien creó la obra y la cite adecuadamente, algo fundamental durante el proceso formativo que transcurre en la universidad.

Las normas internacionales de la ACRL/ALA, CAUL/Anziil y Sconul, así como las nacionales adoptadas por Rebiun [que son utilizadas por las BUs para establecer, implementar, desarrollar, evaluar o mejorar la enseñanza y adquisición de competencias informacionales, y los modelos y marcos a seguir], tienen en cuenta la importancia de utilizar la información de forma ética y legal.

http://www.alfared.org/page/bibliotecas-universitarias/ 570

Nos podemos plantear si las webs de las BUs españolas deben incluir información sobre plagio, con preguntas como:

- ¿Es la biblioteca el sitio más adecuado para situar dicha información?

- ¿Espera el alumno encontrar esa información en la biblioteca?

- ¿Espera el profesor que sea la biblioteca quién tenga la responsabilidad de informar sobre plagio? 
- ¿Ven con buenos ojos las autoridades académicas que sea la biblioteca la que informe sobre plagio?

Si respondiésemos afirmativamente a dichas preguntas, habría que analizar cómo y dónde situar dicha información en la web de la biblioteca. Hay varias posibilidades:

- en la información sobre propiedad intelectual;

- en la información sobre cómo citar y estilos de cita;

- en un tutorial amplio sobre competencias en información o en uno concreto sobre "el uso ético de la información";

- apartado específico y con acceso directo desde la primera página de la biblioteca;

- se puede presentar información textual o incorporar vídeos, tutoriales y material audiovisual atractivo;

- se pueden añadir noticias, artículos, casos conocidos por la prensa o de la propia institución, etc., a modo del dossier realizado por Sureda-Negre y Morey (2008) pero utilizando tecnologías más colaborativas como la que existe sobre alfin en Mendeley o la wiki sobre el estado del arte de la alfabetización informacional en Iberoamérica

http://www.mendeley.com/groups/953381/alfin/papers

\section{http://alfiniberoamerica.wikispaces.com}

Una vez elegidas varias de las opciones propuestas, las bibliotecas que deciden incorporar información sobre plagio realizarán el mismo o parecido trabajo, ya que gran parte de la información es similar y la específica de cada comunidad autónoma o institución es una pequeña parte. Existe la posibilidad de trabajar juntas rentabilizando esfuerzos, siendo más eficientes y logrando informaciones más ricas y exhaustivas. También se puede desarrollar una acción en los consorcios y/o de Rebiun. Parece que Rebiun ha elegido ese camino, y junto con la Comisión Sectorial TIC de la Crue acaba de traducir y adaptar el tutorial de la biblioteca de la University of Sydney "Plagiarism and academic honesty" (El plagio y la honestidad académica). Ya anteriormente había traducido y adaptado el tutorial "Cómo citar y elaborar referencias bibliográficas", de la misma universidad, ambos accesibles desde el portal Cl2 (Competencias informáticas e informacionales). Estos tutoriales pueden ser aprovechados por todas las BUs españolas.

http://www.library.usyd.edu.au/elearning/learn/plagiar ism/index.php http://ci2.es/objetos-de-aprendizaje/tutorial-de-plagio

\begin{tabular}{|c|c|c|}
\hline Biblioteca universitaria & Ubicación en la biblioteca & $\begin{array}{l}\text { Denominación de la información sobre el plagio } \\
\text { y dirección donde se localiza en la biblioteca }\end{array}$ \\
\hline \multirow[t]{2}{*}{ Alcalá } & $\begin{array}{l}\text { Citar la información. } \\
\text { Guía de estilos. Recursos y } \\
\text { ejemplos }\end{array}$ & $\begin{array}{l}\text { Uso ético de la información. El plagio. } \\
\text { http://www2.uah.es/bibliotecaformacion/BECO/plagio/index.html }\end{array}$ \\
\hline & $\begin{array}{l}\text { Propiedad intelectual y derechos } \\
\text { de autor }\end{array}$ & $\begin{array}{l}\text { IV. Plagio. } \\
\text { http://www.uah.es/biblioteca/ayuda_formacion/plagio.html }\end{array}$ \\
\hline Alicante & Propiedad intelectual & $\begin{array}{l}\text { Aprende sobre el plagio y cómo evitarlo. } \\
\text { http://biblioteca.ua.es/es/propiedad-intelectual/aprende-sobre-el-plagio-y-como- } \\
\text { evitarlo.html }\end{array}$ \\
\hline Carlos III de Madrid & Trabajo de fin de grado & $\begin{array}{l}\text { Evitar el plagio. } \\
\text { http://wWw.uc3m.es/portal/page/portal/biblioteca/aprende_usar/TFG } \\
\text { "...evitar utilizar el trabajo de otra persona como si fuera propio..." }\end{array}$ \\
\hline Coruña & Como detectar plaxios & $\begin{array}{l}\text { Copionic: ferramenta gratuíta para comparar documentos en internet. } \\
\text { http://www.udc.es/biblioteca/galego/recestudi.htm }\end{array}$ \\
\hline $\begin{array}{l}\text { Europea Miguel de } \\
\text { Cervantes }\end{array}$ & $\begin{array}{l}\text { Tutorial sobre competencias } \\
\text { informacionales }\end{array}$ & $\begin{array}{l}\text { Comunicar la información. “...necesitas seguir unas pautas para evitar el plagio, } \\
\text { haciendo así un uso ético de la información..." } \\
\text { http://www.uemc.es/es/Biblioteca/Investigacion/Documents/Tutorial\%20sobre\%20C } \\
\text { ompetencias\%20Informacionales.pdf }\end{array}$ \\
\hline Granada & Servicios de la biblioteca & $\begin{array}{l}\text { Ephorus, programa antiplagio. Seminarios formativos. } \\
\text { http://biblioteca.ugr.es/pages/servicios/ephorus }\end{array}$ \\
\hline \multirow[b]{2}{*}{ UNED } & $\begin{array}{l}\text { Uso ético de la información y } \\
\text { citas bibliográficas }\end{array}$ & $\begin{array}{l}\text { Uso ético de la información. } \\
\text { http://www.uned.es/biblioteca/tutorial_uso_etico/presentacion.htm }\end{array}$ \\
\hline & $\begin{array}{l}\text { Competencias genéricas en } \\
\text { información }\end{array}$ & $\begin{array}{l}\text { Módulo 5: Plagio y derecho de cita. } \\
\text { http://ocw.innova.uned.es/competencias_BCA/curso/contenidos/html/modulo5/ } \\
\text { pes_7.html }\end{array}$ \\
\hline \multirow{2}{*}{ Politècnica de Catalunya } & La biblioteca t'ajuda a & $\begin{array}{l}\text { Com evitar el plagi, com detectar-lo. } \\
\text { http://bibliotecnica.upc.edu/es/content/como-evitar-el-plagio-como-detectarlo }\end{array}$ \\
\hline & $\begin{array}{l}\text { InForma't } \\
\text { Ús ètic de la informació }\end{array}$ & $\begin{array}{l}\text { El plagi, què és i com s'evita. } \\
\text { http://bibliotecnica.upc.edu/habilitats/el-plagi-que-es-i-com-sevita-0 }\end{array}$ \\
\hline Politècnica de València & Guías y ayudas & 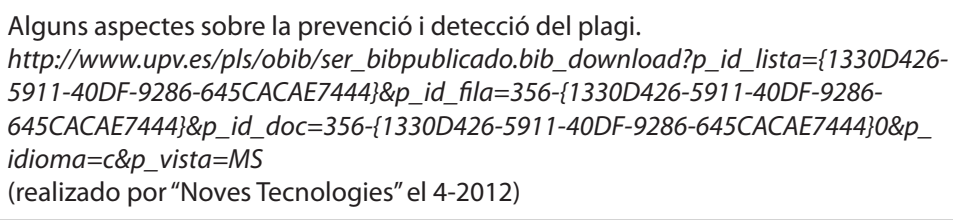 \\
\hline Sevilla & $\begin{array}{l}\text { Formación } \rightarrow \text { Ayudas para } \\
\text { la elaboración del trabajo } \\
\text { académico y/o fin de grado }\end{array}$ & $\begin{array}{l}\text { Evitar el plagio. Guía sobre el plagio académico: tutorial sobre el "Uso ético de la } \\
\text { información". } \\
\text { http://bib.us.es/ximdex/guias/plagio/index.htm }\end{array}$ \\
\hline
\end{tabular}


http://ci2.es/objetos-de-aprendizaje/elaborando-referen cias-bibliograficas

La tabla 1 recoge BUs que en junio de 2012 cuentan con información en sus páginas web, indicando su ubicación y denominación.

\section{Conclusión}

Podemos afirmar que existen razones justificadas para que la biblioteca universitaria incluya en su web información sobre plagio. Además podría ser interesante y estratégico, pues da respuesta principalmente a dos realidades:

- El marco del EEES y su metodología docente, en la que se exige al alumno un abundante número de trabajos a lo largo de sus estudios y una autonomía en su aprendizaje.

- Informar y formar al alumno en el uso ético de la información durante la formación en "competencias informacionales".

La biblioteca universitaria tiene la oportunidad de conseguir que en su comunidad exista un conocimiento claro y materiales de autoformación interesantes y efectivos que logren erradicar en lo posible el problema del plagio en las aulas y cultivar la cultura de la honestidad y el esfuerzo, fomentando la innovación y la creatividad para lograr el verdadero conocimiento que debe impregnar la formación del alumno en su paso por la universidad.

\section{Bibliografía}

Beléndez-Vázquez, Marina; Comas-Forgas, Rubén; Martín-Llaguno, Marta; Muñoz-González, Antonio; Topa-Cantisano, Gabriela. "Plagio y otras prácticas académicamente incorrectas entre el alumnado universitario de nuevo ingreso". IX Jornadas Redes de investigación en docencia universitaria, 16-17 junio 2011. Universidad de Alicante.

http://web.ua.es/es/ice/jornadas-redes-2011/documentos/ posters/184404.pdf

Blanch-Mur, Carles; Rey-Abella, Ferran; Folch-Soler, Andreu. "Nivel de conducta académica deshonesta entre los estudiantes de una escuela de ciencias de la salud". Enfermería clínica, 2006, v. 16, n. 2, pp. 57-61.

Caldevilla-Domínguez, David. "Internet como fuente de información para el alumnado universitario". Cuadernos de documentación multimedia, v. 21, 2010a.

http://multidoc.rediris.es/cdm2/index.php/revista/article/ viewFile/55/57

Caldevilla-Domínguez, David. "Textos sobre plagio, citaciones de trabajos ajenos y declaración de aceptación de renuncia al plagio". Bitácora de David Caldevilla-Domínguez, 2010b.

http://www.ucm.es/info/vivataca/BlogDavid/Plagio. html\#normas

Cavanillas, Santiago. "El ciberplagio en la normativa universitaria". Digithum, 2008, diciembre, n. 10, pp. 1-6.

http://redalyc.uaemex. $m x / s r c /$ inicio/ArtPdfRed.jsp?iCve= 55001006

Comas-Forgas, Rubén; Sureda-Negre, Jaume; Casero, An- tonio; Morey, Mercè. "Las causas del plagio académico entre el alumnado universitario según el profesorado". Revista iberoamericana de educación, 2009, n. 50, pp. 197-220. http://www.rieoei.org/rie50a10.pdf

Comas-Forgas, Rubén; Sureda-Negre, Jaume; Casero, Antonio; Morey, Mercè. "La integridad académica entre el alumnado universitario español". Estudios pedagógicos (Valdivia), 2011b, v. 37, n. 1, pp. 225-225.

http://www.scielo.cl/scielo.php?pid=S0718-070520110001 00011\&script=sci_arttext

Comas-Forgas, Rubén; Sureda-Negre, Jaume; Oliver-Trobat, Miquel. "Prácticas de citación y plagio académico en la elaboración textual del alumnado universitario". TESI (Teoría de la educación, educación y cultura en la sociedad de la información), 2011, v. 12, n. 1, pp. 359-385.

http://campus.usal.es/ revistas_trabajo/index.php/revista tesi/article/view/7837/7863

Comas-Forgas, Rubén; Sureda-Negre, Jaume; Pastor, Miquel; Morey, Mercè. "La búsqueda de información con fines académicos entre el alumnado universitario". Revista española de documentación científica, 2011a, v. 34, n. 1, pp. 44-64.

http://redc.revistas.csic.es/index.php/redc/article/view/ 682/756

Crue/Rebiun. III Jornadas de trabajo de responsables de alfin en las bibliotecas universitarias españolas. Universidad Complutense de Madrid, 9 de abril de 2011.

http://www.rebiun.org/opencms/opencms/handle404? exporturi=/export/sites/Rebiun/Resu_3JALFIN.pdf

e-España. Informe anual sobre el desarrollo de la sociedad de la información en España, 2011. Fundación Orange. http://www.informeeespana.es/docs/eE2011.pdf

Morató-Agrafojo, Yolanda. "Una reflexión necesaria sobre el plagio en el EEES". Revista UPO innova, 2012, v. 1, pp. 361368.

http://www.upo.es/revistas/index.php/upoinnova/article/ view/113/108

Salaway, Gail; Caruso, Judy. "The ECAR study of undergraduate students and information technology". Educause, 2008. http://www.educause.edu/ers0808

Six Degrés. Los usos de internet en la educación superior: de la documentación al plagio. (Encuesta en colaboración con la Universidad de Barcelona y la de Zaragoza), 2008.

http://www.compilatio.net/files/080521_sixdegres-univbarcelona_univ-zaragoza_encuesta-plagio.pdf

Sureda-Negre, Jaume; Comas-Forgas, Rubén. Internet como fuente de documentación académica entre estudiantes universitarios. Palma de Mallorca: Fundación Universidad Empresa de las Islas Baleares, 2006. ISBN: 8469012703 http://xarxasegura.net/descarga/Cerques\%20a\%20Inter net-1.pdf

Sureda-Negre, Jaume; Comas-Forgas, Rubén. "El plagio y otras formas de deshonestidad académica entre el alumnado de la Universitat de les Illes Balears. Resultados generales". Informe de investigación del Grupo Educación y Ciudadanía. Palma: Universitat de les Illes Balears. Departamento 
de Pedagogía Aplicada y Psicología de la Educación, 2008. http://www.ciberplagio.com/universidad/attachment. php?key=37

Sureda-Negre, Jaume; Morey, Mercè. “Cyberplagiarism webliography. References to academic cyberplagiarism on the internet". En: Comas-Forgas, Rubén; Sureda-Negre, Jaume (coords.). Dossier "Academic cyberplagiarism", Digithum.
The humanities in the digital era, n. 10. Universitat Oberta de Catalunya (UOC), n. 10, 2008.

http://www.uoc.edu/digithum/10/dt/eng/sureda_comas_ morey.pdf

Urbina-Ramírez, Santos. 'Ciberplagio: 'construyendo' trabajos universitarios". Edutec, 2004.

http://edutec2004.Imi.ub.es/pdf/159.pdf

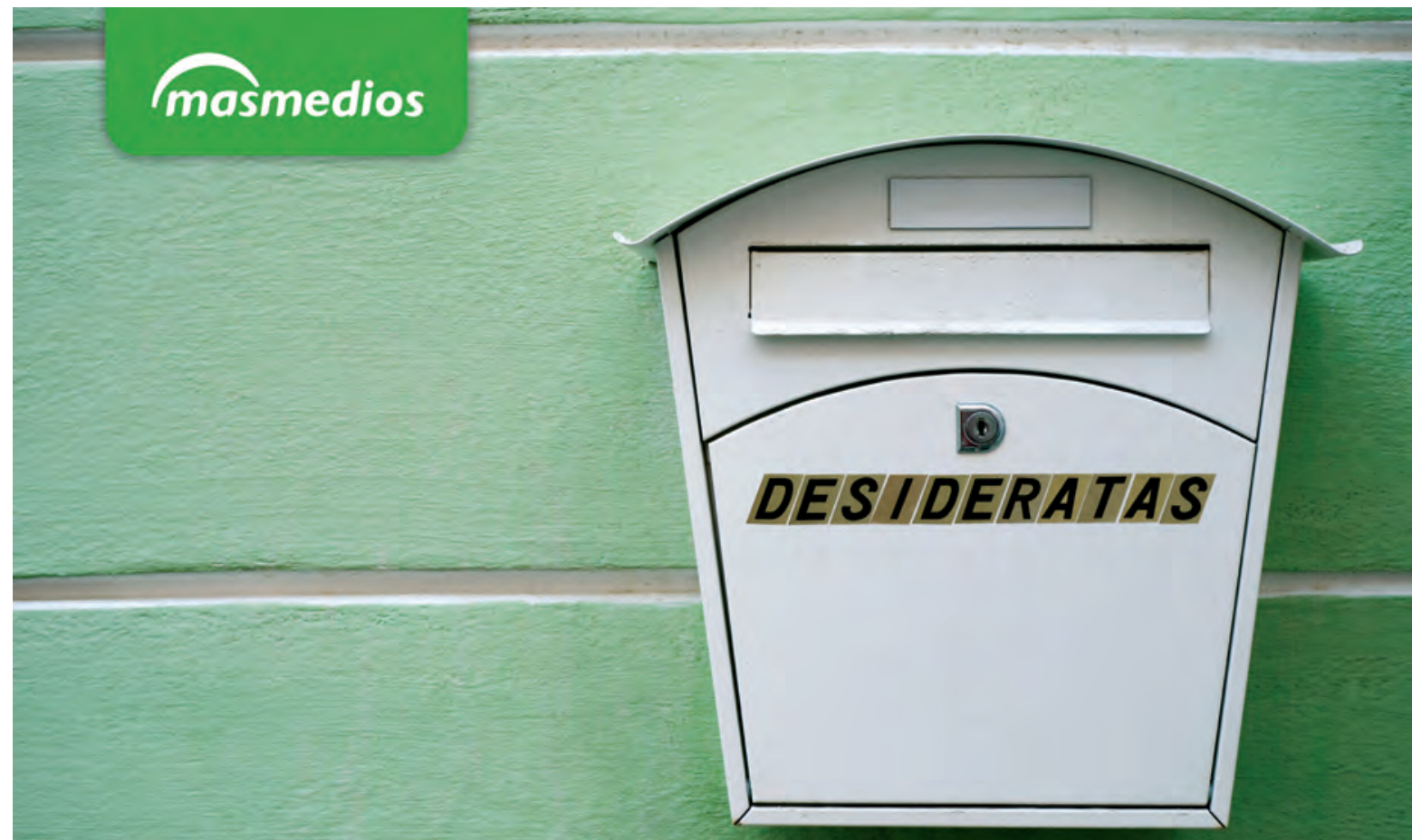

\section{Te entendemos 99}

Sistemas de gestión de bibliotecas Open Source

Interfaces interactivas y OPACs

Repositorios OAI

Gestión documental y de archivos

Digitalización

Outsourcing de servicios documentales

Desarrollo de sitios web / multimedia / e-learning

Comunicación y e-marketing de servicios de información
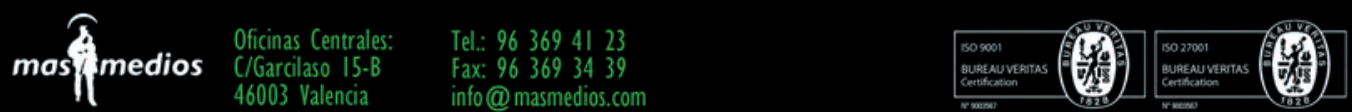

www. masmedios.com 